\title{
Description of Kampo medicines in the clinical practice guidelines for irritable bowel syndrome
}

\author{
Yoshiharu Motoo ${ }^{1}$
}

Received: 23 March 2015/Accepted: 23 March 2015/Published online: 23 April 2015

(C) Springer Japan 2015

The recent article by Fukudo et al. [1] was based on the clinical practice guidelines (CPGs) for irritable bowel syndrome (IBS) [2] published in April, 2014 in Japanese. The evidence-based medicine (EBM) committee of Japan Society for Oriental Medicine (JSOM) has searched the descriptions of Kampo medicines in Japanese CPGs, and has published the results online as the "Clinical practice guidelines containing Kampo products in Japan (KCPG)" (http://www.jsom.or.jp/medical/ebm/cpg/index.html). Since I have been involved in this activity, I regard the clinical question (CQ) "Are Kampo agents effective in treating IBS?" in this article, to be of great interest.

After I read this article [1], I referred to the Japanese CPG [2] in order to obtain more details. Although the ICHUSHI, a Japanese database, was searched, only randomized controlled trials (RCTs) published in English were used as citations for this CQ. However, RCTs on Kampo medicines, published in Japanese, can be used as clinical evidence. In fact, there are two RCTs [3, 4] in Japanese on Kampo medicines for IBS. Reference [4] in particular is a double-blind, placebo-controlled RCT cited in the ICHUSHI. Because the authors searching the databases with keywords such as "herbal medicine" did not find RCTs in English on Kampo medicine for IBS, they could have concluded that there had been no recommendable information on Kampo medicines, instead of searching other herbal medicines.

This comment refers to the article available at doi:10.1007/s00535014-1017-0.

Yoshiharu Motoo

motoo@kanazawa-med.ac.jp

1 Department of Medical Oncology, Kanazawa Medical University, Ishikawa, Japan
This CQ places Kampo medicines in the same category as Chinese or Western herbal medicines, and the references to Kampo medicines were actually articles of Chinese medicines in English. This misleading description might cause overseas readers of this journal to doubt the quality of Japanese Kampo medicines. "Kampo agents" should be defined clearly in the CPG as Kampo products clinically used in Japan.

The EBM committee of the JSOM has compiled RCTs on Kampo medicines online as "Evidence Reports on Kampo Treatment (EKAT)" (http://www.jsom.or.jp/medi cal/ebm/ere/index.html). The above two RCTs in Japanese are also included in the EKAT. I would like to recommend that the CPG developers use the EKAT as a database search engine for the identification of RCTs on Kampo.

Conflict of interest The author declares that he has no conflict of interest.

\section{References}

1. Fukudo $\mathrm{S}$, Kaneko H, Akiho $\mathrm{H}$, et al. Evidence-based clinical practice guidelines for irritable bowel syndrome. J Gastroenterol. 2015;50(1):11-30.

2. Japanese Society of Gastroenterology: clinical practice guideline for functional gastrointestinal diseases 2014: irritable bowel syndrome. In: Sugano K, editor, Nankodo, Co. Ltd., Tokyo; 2014 (in Japanese).

3. Ishii F, lizuka B, Nagasako K, et al. Evaluations of the therapeutic efficacy of Saikokeishito (TJ-10) versus Keishikashakuyakuto (TJ60) for irritable bowel syndrome and Saireito (TJ-114) for ulcerative colitis. Prog Med. 1993;13:2893-900 (in Japanese).

4. Sasaki D, Uehara A, Hiwatashi N, et al. Clinical efficacy of keishikashakuyakuto for irritable bowel syndrome-a multicenter, randomized, parallel-group clinical trial-Rinsho to Kenkyu. Jpn J Clin Exp Med. 1998;75:1136-52 (in Japanese). 